\title{
Applications of Focused Ion Beam Using FEI DualBeam DB235: How Deep Is How Small a Hole? \& How to Drill It Deeper \& Smaller?
}

\author{
Warren J. MoberlyChan*, Erik J. Sanchez**, Peter R. H. Stark***, and John T. Krug**** \\ *Harvard University, Center for Imaging \& Mesoscale Structures, Cambridge, MA 02138. \\ **Portland State University, Department of Physics, Portland, OR 97207. \\ ***Harvard Med. School, Dept of Biochemistry \& Molecular Pharmacology, Cambridge,MA 02141 \\ ****Harvard University, Dept. of Chemistry \& Chemical Biology, Cambridge, MA 02138.
}

With the development of the DualBeam FIB/SEM, it is now possible to resolve the differences at the edges between a small hole and a smaller hole. Whereas a stand-alone FIB will not resolve finer than the features it is processing; the DualBeam enables the important in situ metrology while processing. Machining a small device is not as important as machining two small devices with (exactly) the same geometries and properties. Since spatially discriminating processing tools will always be pushed to their resolution limits, the addition of a $2^{\text {nd }}$ beam of higher resolution to monitor the primary processing beam will enable the actions of the primary beam to become more precise.

There are 2 requests by FIB customers: How small a hole can I make? How deep a hole can I make? But the questions are better worded: How uniform are the edges around a small hole? How deep is a small hole? And how abrupt are the edges of a small hole? (A deep hole will never have the same diameter at the top as at the bottom.) Small holes have potential uses ranging from subwavelength light sources [1], to pores for transcription of DNA molecules [2]. Arrays of small holes in a metal film have enabled light tunneling via enhanced plasmon propagation [3].

In this work the FIB drills small holes through thin membranes, and the holes are observed by the FIB, Field Emission SEM, and TEM. Figure 1a is an SEM image of a 5-by-5 varying array of holes in an amorphous carbon membrane; Figure $1 \mathrm{~b}$ is a FIB image of a 2-by-5 array of holes in $\mathrm{SiO}$. TEM (Figure 2) of the holes confirms that some holes do not go all the way through the membranes. Quantifying the TEM intensity (Figure 2c, 2d) profiles the hole shapes. The FIB can be focused to a $<10 \mathrm{~nm}$ probe, however, the tails of this probe cause much etching outside the probe width. (In this work, it is found that scanning the probe over a small area enables better control of hole formation.)

When considering holes with large depth-to-diameter aspect ratios, a similarity exists to holes drilled for oil. Although telescoping ( $\sim 3 \mathrm{ft}$ diameter at the top and $>4-6$ inches at the bottom) they often are $>20000 \mathrm{ft}$ deep. Important considerations are removal of hole debris without allowing blowouts and steps of refilling and redrilling to shore up the sides of the holes [4]. In a manner somewhat akin, the FIB has been used to partially close (to $<2 \mathrm{~nm}$ ), a big hole previously made by a FIB [2], which then can be again re-FIBed. The FIB and/or the electron beam (EBAD [5]) can be used to assist in localized deposition, (see Fig. 3), which in turn may enable reFIBing with sharper edge retention.

\section{References}

[1] P Stark, D. Larson, R. C. Moore, Prog. ElectroMag. Res. Symp., Boston, (2002).

[2] J. Li, D. Stein, C. McMullan, D. Branton, M. Aziz, J. Golovchenko, Nature, V412, (2001) 166.

[3] S. A. Darmanyan \& Anatoly V. Zayats, Phys. Rev. B 67, (2003) 035424.

[4] J. Trantham \& W. Moberly, "Blowout Prevention", Internal Rept., Keydril Inc., Houston,(1979).

[5] E. J. Sánchez, J. T. Krug, and S. X. Xie, Chem. Phys, V284, (2002) 423. 

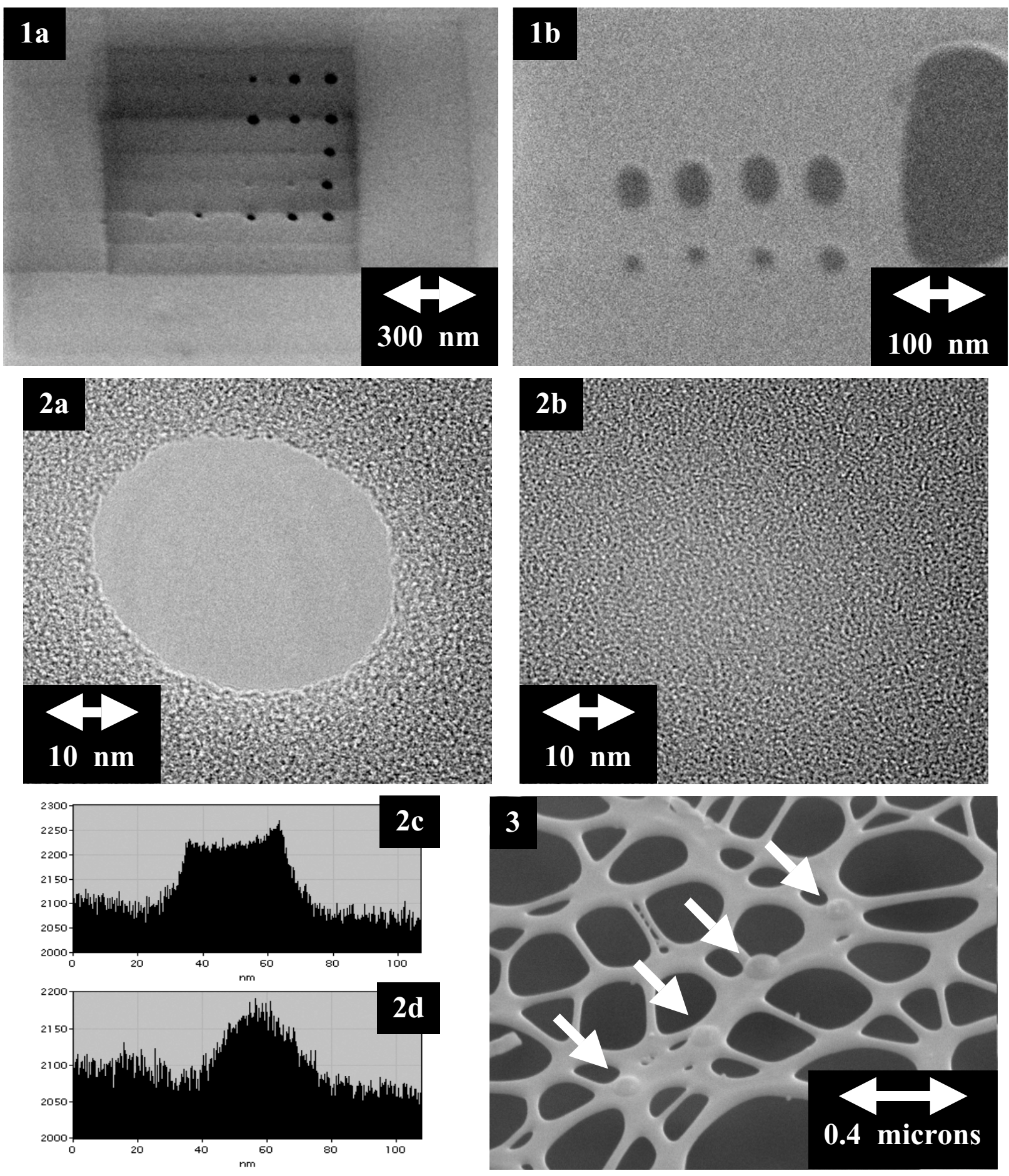

FIG. 1a. SEM image ( $52^{\circ}$ tilt) of FIB-processed 5-by-5 array of holes through thin carbon. FIG. 1b. FIB image ( $0^{\circ}$ tilt) of $2-b y-5$ array of holes through a thin $\mathrm{SiO}$ membrane.

FIG. 2a, 2b. TEM images of hole through carbon membrane, and hole not completely through.

FIG. 2c, 2d. Intensity profiles of TEM images to determine contour of hole shape.

FIG. 3. SEM image of holey carbon TEM grid with islands of SiO2 grown by FIB. Since FIB can also etch while depositing, care is needed to prevent FIBing away the holey carbon before deposition starts. Another method is to initially deposit with electron beam assistance (EBAD [5]). 